\title{
Téoros
}

Revue de recherche en tourisme

\section{Du statut d'entreprise, voire de commerçant, des associations de tourisme}

\section{En droit français et en droit québécois}

\section{Louis Jolin}

Volume 17, numéro 3, automne 1998

Pratiques contemporaines de gestion dans le domaine du tourisme

URI : https://id.erudit.org/iderudit/1072176ar

DOI : https://doi.org/10.7202/1072176ar

Aller au sommaire du numéro

Éditeur(s)

Université du Québec à Montréal

ISSN

0712-8657 (imprimé)

1923-2705 (numérique)

Découvrir la revue

Citer cet article

Jolin, L. (1998). Du statut d'entreprise, voire de commerçant, des associations de tourisme : en droit français et en droit québécois. Téoros, 17(3), 30-36. https://doi.org/10.7202/1072176ar d'utilisation que vous pouvez consulter en ligne. 


\section{A N A L Y 5 I}

\section{DU STATUT D'ENTREPRISE, VOIRE DE COMMERÇANT,}

DES ASSOCIATIONS DE TOURISME'

\section{EN DROIT FRANÇAIS ET EN DROIT QUÉBÉCOIS}

\section{Louis Jolin}

Plusieurs activités touristiques sont réalisées par des organismes sans but lucratif de droit prive ou, plus exactement, par des associations. Dans le secteur du tourisme, on trouve trois grands types d'associations :

- Les associations de promotion, d'accueil et de développement touristiques (au Québec, les offices de tourisme, les bureaux de tourisme et de congrès, les associations touristiques régionales... ; en France, les syndicats d'initiative, les offices de tourisme, les comités départementaux de tourisme, les conseils régionaux de tourisme...) :

- Les associations productrices et gestionnaires de services touristiques qui gèrent des activités et des services touristiques (voyages et séjours de vacances, camps de vacances, villages de vacances, bases et centres de plein air, activités physiques de pleine nature, etc.);

- Les associations professionnelles qui regroupent des membres d'une même profession ou d'un même secteur (les hôteliers, les agents de voyages, les transporteurs, les restaurateurs ont créé des associations pour défendre et promouvoir leurs intérêts).

Trop souvent, on oppose la notion d'association (par définition, sans but lucratif) à celle d'entreprise. Et pourtant, malgré quelques errements de la jurisprudence, en droit québécois comme en droit français, rien n'interdit à une association de tourisme d'exercer une activité économique et même une activité commerciale. Le caractère sans but lucratif ne $\mathrm{s}^{\prime}$ adresse qu'aux membres de l'association qui ne peuvent partager les bénéfices. S'il est accessoire aux objets et aux buts de l'association, l'objet économique, voire commercial, n'invalide pas la compatibilité ; par contre, s'il est prépondérant, il peut se heurter à la capacité limitée de l'association personnifiée, constituée en vertu de la Loi sur les compagnies ou de la Loi sur les corporations canadiennes ${ }^{2}$. L'article 218 de la Loi sur les compagnies mentionne que les lettres patentes peuvent être accordées a dans un but national, patriotique, religieux, philanthropique, charitable, scientifique, artistique, social, professionnel, athlétique, sportif ou autre du même genre ». L'article 154 de la Loi sur les corporations canadiennes est explicite de la même manière. Par contre, la définition du contrat d'association, à l'article 2186 du nouveau Code civil du Québec, est beaucoup plus large encore : « Le contrat d'association est celui par lequel les parties conviennent de poursuivre un but commun autre que la réalisation de bénéfices pécuniaires à partager entre les membres de l'association $\%$.

En droit français, une association, selon

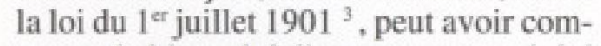
me seul objet celui d'exercer une activité lucrative et commerciale, mais où est l'intérêt si l'on ne peut partager les bénéfices ou si l'activité ne sert pas à l'atteinte des objets sociaux ? La lutte contre le paracommercialisme oblige néanmoins les associations françaises qui exercent une activité commerciale habituelle à le mentionner dans leurs statuts.
Évidemment, c'est une chose de reconnaître la capacité juridique d'une association de conduire une activité économique et commerciale, mais c'est une autre de la considérer comme une entreprise et même comme un commerçant. Le présent article vise à démontrer que, dans certaines conditions et en tenant compte des dispositions propres au droit québécois et au droit français, l'association de tourisme peut être considérée comme une véritable entreprise, voire comme un commerçant. Cette démonstration n'a pas que valeur académique : elle peut avoir des conséquences éminemment pratiques pour des associations qui sont actuellement exclues de certains programmes du fait qu'elle ne soient pas perçues comme des entreprises. Un exemple parmi tant d'autres : même si l'article 14 de la loi constituant le Fonds de solidarité des travailleurs du Québec (F.T.Q.) ${ }^{4}$ définit * l'entreprise comme une société ou une personne morale poursuivant des fins économiques os et prévoit qu'un investissement par le Fonds peut être accordé à une entreprise « sous forme de prêtt, de garantie, de cautionnement, de participation au capital-actions ou autrement », il n"y a eu jusqu'à maintenant aucun investissement dans des associations de tourisme, sous prétexte qu'elles sont sans but lucratif ou qu'elles ne sont pas des entreprises.

\section{UNE DÉFINITION DE L'ENTREPRISE}

Tout en restant un groupement civil sujet à la compétence des tribunaux civils, l'association peut néanmoins être considérée comme une entreprise. La Cour d'appel de Paris définit l'entreprise au sens économique du terme comme étant * la réunion 
en un lieu unique de moyens matériels et humains coordonnés et organisés en vue de la réalisation d'un objectif déterminé ${ }^{5} \%$. Dans son commentaire, le professeur Bolze souligne que si l'adjectif économique ne figure pas dans le texte de l'arrêt rapporté à la suite du terme objectif, \& il convient de l'y ajouter pour préciser à la fois l'étendue et la limite de la notion $\mathrm{d}^{+}$entreprise ${ }^{6} \%$. Pour M. Bolze, la « définition ainsi proposée efface les distinctions classiques entre droit commercial et droit civil, groupements à but lucratif et groupements sans but lucratif, sociétés publiques et sociétés privées : dès lors qu'elles exercent leurs activités dans $u n$ but économique, les entités en cause exercent une activité d'entreprise et sont des entreprises $\%$.

Bien que de plus en plus utilisé par le législateur ou par les juges ${ }^{7}$, le terme entreprise n'est pas comme tel une notion juridique en droit français ; $\approx$ l'entreprise ne jouit pas de la personnalité juridique, à l'instar des groupements reconnus par notre droit : les sociétés, les associations, les G.I.E ${ }^{8}$ \& Certains auteurs veulent reconnaître l'entreprise ès qualité en droit positif, d'autres s'y opposent farouchement ${ }^{9}$. Aux auteurs qui semblent considérer l'entreprise comme une personne s'opposent ceux quỉ la réduisent à un bien appartenant à un entrepreneur individuel ou à un groupement. Castro et Alix proposent une synthèse juridico-6conomique : « l'entreprise - concept économique, lieu d'activité et centre d'intérêt selon une approche socio-ćconomique - est un terme collectif désignant, parmi les groupements dotés de la personnalité morale en droit français, ceux dont l'activité économique combine moyens financiers et humains, mis en ouvre par un organe de décision centralisé, en vue d'une fin économique et/ou sociale ${ }^{10} \%$. A partir d'une telle définition, l'association peut sûrement être une véritable entreprise.

Léo Ducharme, dans sa thèse de doctorat sur l'acte de commerce en droit québécois. a consacré plusieurs pages à la notion d'entreprise. Il rappelle les trois éléments nécessaires à $1^{7}$ existence d'une entreprise : l'élément humain qui se manifeste par un acte de volonté et par la personne du chef de l'entreprise, un élément matériel qui consiste dans l'affectation de certains biens à une fin déterminée, et, comme troisiềme élément, la production économique comme fin déterminée et commune, « Cette production économique peut consister dans la production, la fourniture ou la vente de biens ou encore dans la prestation de services ${ }^{11} \mathrm{~b}$. Ducharme poursuit ainsi :

Lorsqu'on étudie le cas des organismes sans but lucratif, whe distinction, croyons-nous, s'impose entre d'une part, les organismes sans but lucratif à vocation economique et les organismes qui n'ont ni vocation économique et ni but lucratif. Par organisme ayant une vocation économique, nous entendons des organismes ayant pour objet la production de biens ou de services. A l'inverse, les organismes sans vocation économique sont les organismes ayant pour objet des fins religieuses, charitables, patriotiques, ou encore professsionnelles. Les organismes sans but lucratif mais à vocation economique constituent, à n'en pas douter, des entreprises é en conséquence le problème de leur caractere civil ou commercial se soulève à leur égard ${ }^{12}$

Les termes employés ont leur importance... En droit français, l'ordonnance du $1^{\text {er }}$ décembre 1986 rappelle l'obligation d'une association de prévoir dans ses statuts l'activité habituelle d'offrir des produits à la vente, de les vendre ou de fournir des services. L'activité habituelle se confondrait alors avec l'un des objets, qu'il soit ou non prépondérant. Bolze souligne le nécessaire but économique de l'entreprise ; Sami Castro et Nicole Alix, tout en insistant sur l'activité économique propre à l'entreprise, mentionnent que la fin peut être $\ll$ economique et /ou sociale $\%$. Y at-il contradiction? Pas nécessairement ; l'activité économique exercée de façon habituelle devient objet de l'association par l'obligation de l'indiquer dans les statuts... mais l'association peut poursuivre d'autres objets à caractère social. Une association peut conserver une fin sociale tout en ayant un objet ou un but économique qui lui confere la qualité d'entreprise.

Ducharme rappelle que seules les associations ayant vocation économique peuvent être considếrées comme entreprises et que la vocation économique signifie avoir pour objet la production de biens ou de services. Mais, vu la capacité limitée des associations qui doivent poursuivre, en droit québécois, des objets similaires à ceux énumérés dans la loi constitutive, cet objet ne peut être prépondérant contrairement au droit français qui reconnaît que l'association peut avoir pour unique objet l'activité économique. Ducharme semble exclure a priori les associations ayant pour objet des fins religieuses, charitables, patriotiques ou professionnelles. Ces associations ne peuvent-elles pas exercer de façon habituelle une activité économique et avoir comme objet non prépondérant une activité economique? Il faut logiquement répondre dans l'affirmative. Ducharme les exclut peut-être dans la mesure où, dans son esprit, elles n'auraient pour seul objet que des fins religieuses, charitables, patriotiques, professionnelles... Quoi qu'il en soit, une association - et, bien sûr, une association de tourisme (certaines plus que d'autres selon notre typologie) - est une entreprise si elle exerce une activité économique de façon habituelle, ce qui devrait etre inscrit dans ses objets. Ducharme parle alors d'entreprise sans but lucratif.

\section{DES PROBLÈMES DE TRADUCTION}

Les tribunaux canadiens semblent prima facie avoir une vision plus restrictive de l'entreprise. Aux yeux de certains juges, l'entreprise ne serait qu'à but lucratif. Les vicissitudes du langage et de la traduction semblent expliquer cette situation. Un jugement de la Cour suprême du Canada est révélateur à ce sujet. Dans la cause Le Commissaire régional à l'évaluation et le greffier de la municipalité de la ville de Hearst v. Caisse populaire de Hearst Limité $^{13}$ les juges affirment, dans la version française du jugement, que les activités de l'intimée (une coopérative d'épargne et de crédit) * n'équivalent pas à l'exploitation d'une entreprise $\%$. C'est la traduction française de $\alpha$ amount to carrying on business $\%$. L'intimée a pour objet prépondérant de fournir à ses membres des prêts à taux modéré à des fins de prévoyance et d'activités productives et non pas de faire des profits. En consé quence de quoi, les juges ont raison d'affirmer que l'intimée n'a pas à payer d'impôt commercial à l'égard de l'utilisation de son bien-fonds en vertu de l'article 7 de The Assessment Act ${ }^{14}$, Ce n'est pas parce que l'intimée n'exploite pas une entreprise, mais plutôt parce qu'elle n'exploite pas une entreprise à but lucratif. Le texte original anglais de la loi ontarienne utilise l'expression \& business of a traduit de différentes façons dans sa version française : * exploitation de $*$, « exploitation d'une entreprise de $\diamond$ ou même encore, au paragraphe (f) de l'article $7(1) \approx$ dans le cas de l'exercice des professions ou activités suivantes $\%$. Il est clair que le terme anglais a business * recèle une connotation de profit, de but lucratif. D'ailleurs, 
dans le langage courant, le terme $\ll$ business $*$ est aussi traduit par * affaires commerciales $*$; le mot $*$ entreprise $*$ en français équivaut, quant à lui, au terme « undertaking $*$ en anglais, * entreprise commerciale $»$ equivaut à a business undertaking $*{ }^{15}$. En langage juridique, selon le contexte, le terme $\alpha$ business corporation $*$ correspondrait plutôt à la $\alpha$ société anonyme $\propto$ dans un article fort pertinent sur la francisation à l'aveuglette du droit des corporations d'Antoni Dandonneau. Ce dernier souligne d'ailleurs que l'expression a business coporation s s'oppose en anglais à l'expression * non-profit organisation * et qu'en conséquence, on devrait les traduire respectivement par les termes « société anonyme $»$ et « association ${ }^{16}$.

\section{L'APPORT DU NOUVEAU CODE CIVIL DU QUÉBEC}

Ces problèmes de traduction ne trouventils pas leur résolution dans le nouveau Code civil du Québec ? On y retrouve enfin une définition législative de la notion d'entreprise, définition qui fut réclamée lors des discussions en commission parlementaire sur le projet de loi 125 instituant le Code civil du Québec. En effet, le Code civil remplace la notion de commerçant et d'acte de commerce par la notion d'entre: prise et consacre un régime d'exception non seulement aux affaires commerciales, mais à tout le secteur des activités économiques. Le troisième alinéa de l'article 1525 du nouveau Code civil se lit comme suit: « Constitue 1'exploitation d'une entreprise l'exercice, par une ou plusieurs personnes, d'une activité économique organisé, qu'elle soit ou non à caractère commercial, consistant dans la production ou la réalisation de biens, leur administration ou leur aliénation, ou dans la prestation de services $w$.

Il s'agit plus en fait d'une définition de \& l'exploitation d'une entreprise que de la définition de la notion même d'entreprise parce que $l^{7}$ entreprise n'est pas comme telle sujet de droit ; cependant, diverses personnes peuvent revêtir la qualité d'entreprise dans la mesure où elles exercent une activité économique organisée selon les termes de l'article 1525.

L'association de tourisme peut être considérée, en droit français comme en droit québécois, comme une entreprise sans être disqualifiée en sociétế lorsqu'elle exerce une activité économique régulière et signi- ficative, voire commerciale, et sans avoir comme objet prépondérant de réaliser des profits. En droit français, rappelons-le, l'association peut strictement avoir un tel objet prépondérant s'il est prévu dans les stạtuts, mais, comme le souligne fort à propos Sami Castro, a où serait l'intérêt de faire des bénéfices, alors qu'il ne serait possible de les partager ni immédiatement, ni à terme ? ${ }^{17}$. En droit québécois, le contrat de société (article 2186 du C.c.Q.) permet spécifiquement, dans un but lucratif pour les parties au contrat, d'exploiter une entreprise ; le contrat d'association (même article) permet aux parties de poursuivre un but commun a autre que la réalisation de bénéfices pécuniaires à partager entre les membres », ce qui n'exclut pas le fait d'exploiter une entreprise. Le but commun peut-il être l'exploitation d'une entreprise ? Pour une association non personnifiée, rien ne l'interdit ; pour une association dotée de la personnalité juridique en vertu de la Loi sur les compagnies (le cas de figure le plus fréquent), sans doute si l'exploitation de l'entreprise est liée aux objets autorisés par la loi constitutive et, si tel n'est pas le cas, qu'elle ne constitue pas un objet prépondérant.

Un jugement québécois ${ }^{18}$ a déjà « requalifié $*$ en société commerciale une association de commerçants d'un quartier de Montréal à cause des motivations et des activités lucratives de ses membres. Plusieurs commentateurs ont souligné que ce jugement de première instance était mal fondé en droit parce que les membres ne tiraient aucun avantage pécuniaire direct de l'activité même de l'association, mais uniquement des avantages indirects. On trouve aussi de telles erreurs (reprochées par les commentateurs) dans quelques jugements français qui, même en l'absence de tout partage de bénéfices, ont « requalifié $*$ une association en société de fait ${ }^{19}$.

Mais ces jugements sont l'exception : l'association peut donc être une entreprise sans risque de requalification en société si les principes de base sont respectés. Cette association-entreprise peut-elle maintenant être qualifiée de commerciale ou même de commerçante ? Dans l'affirmative, à quelles conditions ?

\section{UN GROUPEMENT COMMERCIAL}

Être ou ne pas être commerçant ? Cette question se pose, bien sûr, lors de l'exercice habituel d'une activité commerciale, mais la réponse n'est pas évidente, encore moins lorsqu'il s'agit d'une association. Si l'on hésite encore à accorder à l'association le statut de commerçant, il reste que l'association sera considérée sous certaines conditions comme un groupement commercial.

Pour qualifier une association de commerciale, d'entreprise commerciale ou de groupement commercial par opposition au groupement civil, ce qui entraîne l'application de règles du droit commercial, il y a cependant une différence $\mathrm{d}^{\dagger}$ approche entre le droit québécois et le droit français, principalement à cause de la théorie des objets corporatifs (ou celle de l'ultra vires) toujours en vigueur en droit québécois.

\section{LE DROIT FRANÇAIS}

Le droit français semble accorder la primauté au fait même d'exercer habituellement et régulièrement une activité commerciale quels que soient les objets de l'association ; si l'activité commerciale n'est pas une simple activitế accessoire, la qualification d'association commerciale s'impose. Cette interprétation a d'autant plus cours en droit físcal. La célèbre affaire de l'Institut musulman de la mosquée de Paris ${ }^{20}$ sert de phare en la matière: l'association qui exploite un magasin de vente de détail de viande à but lucratif est soumise aux règles de preuve du droit commercial ; sans avoir accordé explicitement la qualité de commerçant à cette association, la Cour de cassation ne l'a pas moins considérée comme groupement commercial sujet aux règles du droit commercial. Plusieurs autres associations se sont vues reconnaître la qualité d'association commerciale du fait d'accomplir sur une base régulière des opérations commerciales ${ }^{21}$. Cependant, la Cour de cassation, dans l'affaire du Club du Vert Galant, a introduit un autre critère : il faut que cette activité ait un caractère spéculatif répété au point de primer l'objet statutaire (qui serait bien sûr de caractère civil) : c'est la réhabilitation de l'objet statutaire mais qui sera analysé en fonction de l'activité civile de l"association.

Les associations de tourisme, plus particulierrement les associations productrices et gestionnaires de services touristiques, s'exposent à l'application du droit commercial, mais là aussi, il faut que le caractère spéculatif de l'activité soit nettement évident : il se révèle si l'association pros- 
pecte sa clientèle sur une grande échelle, en dehors de ses propres adhérents ${ }^{22}$. Qu'en est-il alors des associations de tourisme agréées en vertu des dispositions du 11 juillet 1975 et, dorénavant, de la Loi du 13 juillet 1992 relative à l'organisation de voyages et de séjour? Ces associations ne peuvent effectuer \& les opérations mentionnées à l'article premier qu'en faveur de leurs membres *, ni * diffuser, à l'adresse d'autres personnes que leurs adhérents ou ressortissants, qu'une information générale sur leurs activités et leurs buts ${ }^{23}$. Donc, même si ces associations accomplissent des actes de commerce, elles semblent exclues du secteur commercial dans la mesure où elles respectent les obligations de l'agrément. Ces associations ont beau avoir pour objet l'organisation et la vente de voyages, ce qui devrait les conduire dans le champ de la commercialité, elles ne seront pas qualifiées de commerciales vu les conditions imposées à l'exercice de leur activité commerciale ${ }^{24}$. Mais si ces obligations ne sont pas respectées, elles peuvent se voir retirer l'agrément ${ }^{25}$ et seront qualifiées de commerciales encore que, dans l'état actuel du droit, elles aboutissent manifestement à un cul-de-sac : en effet, en vertu des articles 4 et 8 , elles ne peuvent obtenir la licence d'agent de voyages.

On peut appliquer le même type de raisonnement aux maisons familiales et aux villages de vacances agréés, tout en se rappelant que l'agrément ne porte pas sur l'association gestionnaire comme telle, mais sur l'établissement. Parmi les conditions de l'agrément d'un village de vacances sans but lucratif, en droit français, on retrouve l'obligation de réserver les services aux adhérents et de ne pas recourir à la publicité commerciale, avec un prix de pension inférieur au plafond ministériel ; la maison familiale de vacances, destinée par définition aux familles ayant un niveau de vie réduit, doit offrir des prix de journée notablement inférieurs à ceux des hôtels ou pensions de familles voisins. Si l'association gestionnaire respecte ces conditions, elle reste certainement en dehors de la commercialité. Dans le cas contraire, les établissements perdront leur agrément et, éventuellement, les subventions qui en découlent, mais les associations pourront quand même exploiter de tels établissements sans agrément et être considérées comme commerciales ${ }^{26}$.

En ce qui concerne les deux autres catégories d'associations de tourisme, elles ne relèvent pas en principe du secteur com- mercial : on n'y trouve généralement pas d'activité à caractère spéculatif au point de primer l'objet statutaire qui est de la nature d'un service public pour les associations de promotion, d'accueil et de développement ; les prestations des associations professionnelles sont surtout de nature corporative, axées sur la représentation et la défense des intérêts des membres. Mais, si d'aventure, une de ces associations avait une importante activité commerciale habituelle et réguliềre, il ne faudrait pas hésiter à la qualifier de groupement commercial avec les conséquences qui s'ensuivent.

\section{LE DROIT QUÉBÉCOIS}

Le droit québécois envisage la question dans une autre perspective pour atteindre des résultats similaires au droit français. Pour Paul Martel : « Même si certains des pouvoirs de la corporation sont ceux d'une compagnie commerciale, ils sont exercés non pour faire un profit, mais pour atteindre les objets sans but lucratif de la corporation. L'exercice de tels pouvoirs 'commerciaux' ne fait pas a priori perdre à la corporation son caractère civil. Il ne faut pas, cependant, que sous le couvert d'objets désintéressés la corporation s'adonne systématiquement à des activités commerciales, comme par exemple la spéculation immobilière $^{27} \%$ La question des objets est primordiale en droit québécois, car les associations personnifiées ont une capacité limitée par des objets prévus dans leurs lettres patentes et dans la loi constitutive ; elles ne peuvent avoir d'autres buts ou objets que s'ils sont analogues à ceux énumérés dans la loi. Tout activité qui ne serait pas compatible avec ces objets pourrait être déclarée ultra vires. Par contre, nous l'avons vu, une association peut néanmoins avoir un objet lucratif pourvu qu'il ne soit pas prépondérant. Rappelons l'affirmation du juge McIntyre dans la cause Caisse populaire de Hearst limitée : « Si l'objet prépondérant est la réalisation de profits, l'activité peut alors être considérée comme une entreprise ${ }^{28}$ \% (en anglais, * business $*$ qui aurait dû être traduit par \& entreprise à but lucratif $»$ ).

$\mathrm{Si}$, a priori, les « pouvoirs commerciaux * de la corporation, pour reprendre l'expression de Martel, ne font pas perdre à l'association personnifiée son caractère civil, a posteriori, il peut en être autrement. S'appuyant sur un jugement de la cour supérieure, Beaubien et Bibeau c. l'Union économique d'habitation ${ }^{29}$, mettant en cause une association incorporée selon la troisième partie de la Loi sur les compagnies et, par analogie, sur des articles de loi concernant les diverses formes de coopératives, sur l'article 2471 du Code civil du Bas-Canada déclarant que « l'assurance mutuelle n'est pas une opération commerciale $n$, sur divers jugements impliquant des mutuelles d'assurance ${ }^{30}$, Léo Ducharme est d'avis qu' une association, pour se maintenir hors du domaine commercial, doit absolument limiter ses activités à ses seuls membres.

« Rien n'empêche, par exemple, une corporation sans but lucratif qui exploite une salle de spectacles ou un centre de ski, de faire affaires avec le public en général et non avec ses seuls membres. Dans un tel cas et dans la mesure où les membres sont appelés à profiter des activités de leur corporation avec les non-membres, 1 'organisme devient, tout comme la mutuelle à primes fixes faisant affaires avec le public, une entreprise commerciale ${ }^{31}$ \%. Si l'association de tourisme exerce une activité commerciale habituelle et régulière qui s'adresse à la fois aux membres et aux non-membres, elle sera considérée comme une association commerciale ; si elle ne s'adresse qu'aux membres, elle devrait, comme en droit français, rester un groupement civil. Contrairement à la loi française de 1992 relative à l'organisation de voyages, la loi québécoise sur les agents de voyages ne fait pas de différence entre une entreprise à but lucratif et une entreprise à but non lucratif pour l'obtention du permis et, pour cette dernière, elle n'impose aucune obligation de s'adresser aux seuls membres et de ne pas faire de publicité commerciale. Il en est de même pour les associations gestionnaires d'établissements de vacances (centres de vacances, camps familiaux, auberges de jeunesse, etc.) qui doivent obtenir un permis en vertu de la Loi sur les établissements touristiques, au même titre que les hôtels à but lucratif, et qui n'ont aucune obligation d'offrir leurs services aux membres seulement. Ne retrouvant aucune présomption dans la loi, il faut alors analyser le comportement effectif de chaque association.

Les associations productrices et gestionnaires de services touristiques, dans la mesure où elles accomplissent des actes de commerce de façon habituelle et régulière auprès du public en général, se verront d'autant plus reconnaître le caractère commercial que la jurisprudence accorde spontanément ce caractère aux entreprises à but lucratif faisant fonction d'inter- 
médiaire, ou dispensant des services de transport, ou encore exploitant un hötel, un restaurant ou un débit de boisson ${ }^{32}$.

\section{DE GROUPEMENT COMMERCIAL À COMMERC̣ANT}

\section{UN PAS A FRANCHIR EN DROIT QUÉBÉCOIS}

Une fois reconnue comme groupement commercial, il n'y a qu' un pas à franchir pour que l'association soit considérée comme commerçante. Il ne semble y a voir aucun obstacle en droit québécois. Au contraire, on ne craint pas d'assimiler explicitement les organismes à but non lucratif aux commerçants dans certaines situations. La Loi sur la protection du consommateur fournit l'exemple le plus probant : cette loi « s'applique à tout contrat conclu entre un consommateur et un commerçant dans le cours de son commerce et ayant pour objet un bien ou un service ${ }^{33} w$ et $1^{7}$ article 3 stipule: * Une corporation qui ne poursuit pas des fins lucratives ne peut invoquer ce fait pour se soustraire à l'application de la présente loi *. Nicole L'Heureux en conclut : la loi déborde la notion de commerçant pour comprendre les corporations sans but lucratif et les autres entreprises qui exercent une activité économique sans but de profit comme les coopératives, les caisses d'épargne et de crédit, les syndicats coopératifs malgré le caractère civil de leur objet $x^{34}$. Mais la rédaction de l'article est toute en nuances : le premier alinéa de l'article 3 stipule que les coopératives, les caisses d'épargne et de crédit et les syndicats coopêratifs sont soumis à l'application de la loi, tandis que le deuxième alinéa mentionne seulement que la corporation qui ne poursuit pas de fins lucratives ne peut invoquer ce fait pour se soustraire à la loi. Encore faut-il cependant que la corporation soit une entreprise commerciale, qu'elle se situe dans le champ de la commercialité : si le fait de ne pas poursuivre des fins lucratives ne peut soustraire un organisme à l'application de la loi, toute association qui transige avec des consommateurs n'exerce pas automatiquement une activité commerciale. Mais ce n'est pas l'interprétation qui a cours actuellement à l'Office de protection du consommateur du Québec qui veut donner la couverture la plus large possible à la Loi sur la protection du consommateur. D'ailleurs, le nouveau Code civil va dans le sens d'une interprétation large en donnant une définition du contrat de consommation qui met en jeu une première partie, le consommateur, étant nécessairement une personne physique, et une deuxième partie, \& laquelle offre de tels biens ou services dans le cadre d'une entreprise qu'elle exploite $x^{35}$. Mais quelle que soit l'interpretation, même la plus restrictive, on ne craint pas de reconnaître la qualité de commerçant à une association personnifiée ou à une corporation sans but lucratif.

En ce qui concerne le statut de commercant, trois auteurs contemporains, Nicole L'Heureux, Albert Bohémier et Pierre-Paul Côté, à partir de la définition de commerçant d'Antonio Perrault : * Est commerçant celui qui fait des opérations commerciales à l'état professionnel $8^{36}$, retiennent les conditions essentielles suivantes: 1faire des operations commerciales ; 2 - à titre de profession habituelle ; 3-faire des opérations commerciales en son nom et pour son compte. (Albert Bohémier et Pierre-Paul Côté ajoutent : ne pas être artisan). Une personne morale agit en son nom propre, pour son compte, lorsqu'elle prend elle-même le risque commercial ; elle exerce profession dans la mesure où elle pose des actes de commerce de façon habituelle. Mais à l'égard des associations personnifiées, plus spécifiquement des corporations constituées selon la partie III de la Loi sur les compagnies, Bohémier et Côté ne tirent pas tout à fait les mêmes conclusions que L'Heureux. Selon Bohémier et Côté, a Dans le cas de personnes morales, on considérera que la compagnie ou société constituée en vertu des lois générales, fédérales ou provinciales, dans un but lucratif (Parties I et IA de la Loi sur les compagnies), est un commerçant alors que celle constituée dans un but non lucratif (but national, patriotique, religieux, philanthropique, artistique ou autre) n'est pas un commerçant ${ }^{37} \mathrm{w}$. L'Heureux, pour sa part, est plus nuancée : * A l'égard des sociétés incorporées en vertu de la première partie de la Loi des compagnies, il existe une présomption absolue de leur qualité de commerçant en raison de leur but lucratif ; elles sont soumises au régime juridique du droit commercial. Il en est de même à l'égard d'une société qui serait incorporée sous la troisième partie de la Loi des compagnies, si elle se livre à des opérations commerciales à but lucratif ${ }^{38}$. L'Heureux rejoint ainsi le point de vue de Ducharme qui, malgré la présomption de non commercialité des entreprises à but non lucratif, reconnaît qu'une telle entreprise puisse être commer- ciale, mais elle s'appuie malheureusement sur un jugement mal fondé en droit qui a * requalifié w erronément une association en société commerciale de fait (Pelletier c. Association du Progrès du Plateau Montroyal inc, mentionne plus haut).

\section{L'OBSTACLE DU REGISTRE DU COMMERCE EN DROIT FRANÇAIS}

S'il est concevable qu'une personne physique puisse exercer simultanément plusieurs activités régulières dont l'une seulement correspond à sa profession, il est difficile de faire cette distinction entre activité commerciale et profession pour une personne morale qui, très souvent, n'a qu'une activité unique (ex. gérer un établissement d'hébergement touristique). Selon Yves Reinhard, a il semble donc qu'une association qui, de façon habituelle, se livre à une activité habituelle devrait être qualifiée de commerçante, au sens de l'article $1^{\mathrm{tr}}$ du code ${ }^{3{ }^{3}}{ }^{3}$. Même si la jurisprudence française hésite à reconnaître explicitement la qualité de commerçant à une association, elle l'a fait au moins implicitement, car la qualité de commerçant est la condition d'application de certaines règles du droit commercial, notamment en matière de preuve ou de redressement judiciaire ${ }^{40}$. La cour d'appel de Colmar a été, quant elle, très explicite, en reconnaissant dans les attendus la qualité de commerçant à une association ${ }^{41}$. Mais comment comprendre la décision de la cour de cassation dans l'affaire Beaufils c. Association Foyer Lelo Lagrange ${ }^{42}$ qui semble refuser à l'association la qualité de commerçant? Par l'effet combiné des dispositions sur le registre du commerce et des sociétés ${ }^{43}$ et de la Loi du 20 mars $1956^{44}$. En effet l'association, n'étant pas inscrite au registre du commerce et des sociétes - il est presque impossible pour les associations de l'être pour le moment ${ }^{45}$ -, ne peut justifier sa qualité de commerçant : l'immatriculation au registre du commerce et des sociétés est une condition pour la concession d'une locationgérance selon l'article 2 de la Loi du 20 mars 1956. Faut-il alors, pour être reconnue comme commerçant, que l'association soit immatriculec au registre du commerce et des sociétés ? Et comme il est impossible pour une association d'être immatriculée, peut-on conclure que si une association peut être effectivement un groupement commercial, elle ne saurait avoir stricto sensu la qualité de commerçant ? Les auteurs ${ }^{\text {th }}$ ne vont pas jusque-là, même si plusieurs souhaitent que l'ambiguité soit 
dissipée. Ils s appuient notamment sur une réponse du Garde des sceaux, le ministre de la Justice, affirmant que la jurisprudence qui reconnaît au moins implicitement la qualité de commerçant aux associations * ne semble pas avoir été remise en cause par l'arrêt de la même chambre du 19 janvier $1988 \%^{47}$. Comme pour la procédure de déclaration en droit québécois, l'immatriculation au registre, qui vise la protection des tiers, ne crérait qu'une présomption de $\mathrm{I}^{+}$intention de faire commerce, mais ne serait pas indispensable à la qualité de commerçant. N'empêche que cette immatriculation est nécessaire pour bénéficier de certains avantages (droit au bail, location-gérance).

\section{QUELQUES CONSÉQUENCES}

Dans un autre article, nous pourrions ćtudier en profondeur les conséquences de certaines opérations sur l'exercice ainsi que sur la fiscalité de la reconnaissance du caractère économique, voire commercial, de l'activité des associations de tourisme.

Leur reconnaissance progressive comme acteurs économiques ou comme entreprises ont amené le législateur français à conférer aux associations certains droits, tout en les soumettant à certaines obligations, au même titre que les autres types d'entreprises. Que ce soit en matière de prévention des difficultés des entreprises, de redressement et de liquidation judiciaire, ou encore de réglementation générale des prix et de la concurrence, qu'il

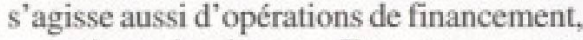
on voit se dessiner en France une nette tendance législative depuis 1967 ; on ne fait plus de distinctions entre l'association et la société, toutes deux étant des entreprises soumises aux mêmes règles ${ }^{48}$.

Le droit québécois des associations n"a pas attendu l'affirmation du caractère 6 conomique de nombreuses associations, ni leur reconnaissance comme entreprises ; par son origine, par ses caractéristiques (d'une certaine maniềre, droit d'exception des compagnies à fonds social), le droit québécois assimile dès le départ les associations aux sociétés anonymes, aux compagnies à fonds social avec les adaptations nécessaires, compte tenu que les associations sont sans but lucratif et qu'elles n'ont pas de capital-actions. Dans le partage de lois s'appliquant ou non aux associations dotées de la personnalité juridique, le législateur a maintenu applicables les articles concernant la prévention des dif- ficultés des entreprises (tenue de livres comptables, nomination obligatoire d'un vérificateur au moins au fédéral...), concernant la faillite et la liquidation des entreprises, la réglementation économique des prix et de la concurrence, le financement par émission d'obligations... ${ }^{4}$ ?

Par contre, il faut nuancer en ce qui concerne le statut de commerçant de l'association de tourisme. L'association de tourisme, considếrée comme commerçante, devrait logiquement être soumise aux règles du droit commercial. En droit québécois, c'est théoriquement le cas, or, depuis l'avènement du nouveau Code civil du Québec, il n'y a plus de régime particulier pour les commerçants mais bien pour toute entreprise qu'elle soit ou non de nature commerciale, qu'elle soit ou non à but lucratif. Une quarantaine d'articles tracent le contour de ce régime pour les personnes qui exploitent une entreprise (articles sur la preuve, la vente de l'entreprise, la présomption de solidarité des débiteurs d'une obligation, une hypothèque concernant l' universalité des biens, etc.) ${ }^{50}$. Sous réserve de quelques exceptions (le secret commercial ou l'affrètement maritime ${ }^{51}$ ), la notion de commerce fait dorénavant place au concept plus large d'entreprise. Il n'y a pas non plus, au Québec, de tribunal de commerce : les tribunaux civils sont compétents en matière civile.

Plusieurs règles commerciales en droit français s'appliquent aux associations commerçantes (elles peuvent être justiciables devant les tribunaux consulaires (de commerce), la preuve par livre de commerce peut être administrée contre elles, les règles de solidarité, de mise en demeure, de prescription leur sont applicables). Deux restrictions cependant : elles ne peuvent, dans l'état actuel du droit français, mettre en location-gérance leur fonds de commerce ni bénéficier des avantages du bail commercial, car elles ne peuvent faire état de l'immatriculation au registre du commerce et des sociétés.

Enfin, au plan fiscal, la situation est fort complexe. Retenons tout simplement que le régime de faveur qui est consenti aux associations sans but lucratif disparait dans la mesure où les associations se situent dans le champ de la commercialité. Cela est d'autant plus vrai en France que la situation fiscale ne dépend pas du statut juridique de l'association, mais de la nature même des activités et de la façon dont elles sont exercées ${ }^{52}$. En droit québécois (et canadien), l'Administration fiscale souhai- terait appliquer un semblable raisonnement mais les tribunaux canadiens semblent maintenir un régime de faveur si les objets de l"association continuent à être de ceux qui justifient le régime de faveur ${ }^{33}$.

Louis Jolin est professeur au Département d'études urbaines er touristiques de l'École des sciences de la gestion de I'UQAM et directeur de Téros.

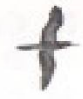

\section{NOTES}

1 Le présent article est une adaptation d'un extrait de sa thèse de doctorat en droit des affaires, soutenue en 1995, à l'Université de Lyon III. Titre de la these : Associations et activités touristiques en droit français at en droil quebecois, sous la direction de $\mathrm{M}$. Gérard Sousi.

2 Loi sur les compagnies, L.R.Q., c. C-38 ; Loi sur les corporations canadiennes, L.R.C., c. C-32.

3 Lol du ler juillet 1901 relative au contrat d'association, J. O., 2 juillet 1901 (plusieurs fois modifiée).

4 Loi constituant le Fonds de solidarité des travailleurs du Québec (F.T.Q.), L.R.Q., c. F3.2 .1 .

5 Cour d'appel de Paris, 28 mai 1986, D. 1987.2 , p. 562 , note C. Bolze

6 Le Premier président Drai de la cour d'appel de Paris aurait confirmé en ce sens la décision rendue et la note du professeur Bolze mềme si l'adjectif "économique ne figure pas dans le texte de l'arrềt rapporté.

7 Quelques lois : la Loi du 25 janvier 1985 sur le redressement et la liquidation judiciaires dissocie l'entreprise de l'entrepreneur dans le but de sauver l'entreprise (cette loi a aussi ćté modifiée par la Loi du 10 juin 1994) : la Loi du 11 juillet 1985 relative à l'entreprise uni-personnelle à responsabilité limitée fait de l'entreprise un sujet de droit.

8 Castro et Alix, 1990: 22 .

9 Voir aussi Ducharme (1996: 85 et \$.).

10 Castro et Alix, $1990 \div 23$

11 Ducharme, $1996: 78-79$.

12 Ducharme, 1996 : 99.

13 The Regional Assessment Commissionner and the Municipal Clerk of Hearst c. Caisse populaire de Hearst Limitée $* 1983 ; 57$.

14 The Assessment Act (ou selon la version française : Loi sur l'évaluation foncière), R.S.O. 1970, chap. 32 .

15 Voir Harrap's Standard French and English Dictionary.

16 Dandonneau, $1978: 89-97$.

17 Castro, 1987:77. 
18 * Pelletier c. Association du progrès du Plateau Mont-Royal inc s., (1966) C.S., p. 370.

19 Quelques exemples: Trib. Com. Rennes, 13 janvier 1978, D. 1979, Inf. Rap. 313, obs. Alaphilippe et Karaquillo, Rew Tr. Dr. Com., 1979. 488, ne 13, obs. Alfandari ; Cass. Crim., 3 janvier $1983, D ., 1984.615$, note T. Renoux.

20 Cass. Com., 17 mars $1981, n^{\circ} 79-14.117$, * Institut musulman de Paris c. Lahoucine *, Rev Soc, 1982, p. 124, obs. Sousi.

21 Cass, Com, 12 février 1985, $\mathrm{n}^{\mathrm{*s}}$ 83-10,864 et 83-11,2886, Bull. Civ. IV, $\mathbf{n}^{\circ} 59$, p. 50.

22 Cass. Com. 8 juillet $1969, \mathrm{n}^{6} 68-10.250$, J.C.P., éd. G. 1970, II, $\mathrm{n}^{0} 16155$ bis, note J.A. ; C.A. Paris, 30 avril 1980 : G.P. 12 février 1981, * Association loisirs et vacances de la jeunesse : ; C.A. Bastia, 26 décembre 1986, Bull. Inf., Cass. $1989, \mathrm{n}^{\circ} 446$, p. 21.

23 Article 8 de la loi $\mathrm{n}^{\circ} 92-645$ du 13 juillet 1992 , J.O., 14 juillet 1992, p. 9457 . Il s'agit d'une rédaction légèrement différente de l'article 7 de la Loi du 11 juillet 1975 , article qui se lisait comme suit : $\alpha$ Les associations, groupements et organismes sans caractère lucratif ne peuvent effectuer les opérations mentionnées à l'article premier qu'en faveur de leurs membres. Ils ne peuvent faire, sous quelque forme que ce soit, a l'adresse d'autres personnes que leurs membres, une publicite se rapportant à des voyages ou des séjours déterminés $\mathrm{x}$

24 C'est ce qui expliquerait vraisemblablement que la procédure de règlement judiciaire appliquée au Touring Club de France ait été entendue par le tribunal de grande instance de Paris et non par le tribunal de commeree: T.G.I. Paris, 12 juin 1981, R.T.D. com. 1981 , p. 561 , obs. Alfandari et Jeantin. Il en serait de meme dans la cause * Tourisme et travail d'Orléans s, qui a vu un tribunal civil se prononcer sur le dépôt de bilan de l'association : C.A. Paris, 18 novembre 1986, R.T.D. com., 1987, p. 78, obs. Alfandari et Jeantin.

25 Article 45 du décret n 94-490 du 15 juin 1994 pris en application de la loi du 13 juillet 1992.

26 Voir Décret $n^{\circ} 75-402,18$ novembre 1975 (Gaz. Pal. 1975, 2, Légis, 742; J,C.P. 1975. III, 43568$)$ relatif aux villages de vacances et les divers exemples recensés dans la circulaire du premier ministre.

27 Martel et Lebel, 1994 : 6-8.1.

28 Déjă citúe.

29 Déjà citée.

30 * The British Empire Mutual Life Insurance c. Bergevin *, (1895) 5 B.R.,p. 55 ; * Mutual Life Assurance Co c. Giguère *, (1902) 32 S.C.R., p. 348 ; * MeNicoll c. Mutual Benefit Health and Accident Association $\%_{4}$ (1961) R.P. p. 334 .

31 Ducharme, 1996: 106-107.

32 Ducharme, 1996: 113-118. $\AA$ titre d'exem. ples, * Ducharme c. Bélair $w_{;}$(1941) $79 \mathrm{Cs}_{\text {, }}$, p. 244 (entreprise de taxi) : * Proulx c.
Beaudoin \%, (1941) 79 C.S., p. 423 (hôtel) : * The National Breweries Ltd. c. Champagne $\%$, (1919) 25 R. $J_{n}$ p. 254 confirmé par (1920) 26 R.L. n.S., p. 224.

33 Article 1 de la Loi sur la protection du consommateur, L.R.Q., c. P-40.1.

34 L'Heureux, $1986: 25-26$.

35 Article 1384 du Code civil du Québec.

36 Perreault, 1936 : 604.

37 Bohémier et Côté, 1985 : 37.

38 L'Heureux, 1975 : 101.

39 Reinhard, Yves, Activité commerciale *, Lamy association, étude 234, Paris, Lamy S.A. p. 17. L'article ler du Code français du commerce mentionne $\alpha$...sont commerçants ceux qui exercent des actes de commerce et en font la profession $\mathrm{s}$.

40 Article 109 du Code du commerce pour la preuve, article 7 de la Loi du 25 janvier 1985 pour la procédure de redressement judiciaire (modifié par l'article 14 de la loi du 10 juin 1994). Cass, com. 17 mars 1981 , n 79 14.117, Rev. Soc, 1982, p. 124, obs. Sousi : Cass. Com., 12 février 1985, $\mathrm{m}^{\mathrm{ms}}$ 83-10.864 et 83-11.286, Bull. Civ. IV, n" 59, p. 50.

41 - Attendu (que les premiers juges) ont conclu a juste titre que l'association faisait des actes de commerce et qu'elle avait donc la qualité de commerçant -. C.A. Colmar, 14 mai 1980 , Rev.soc. 1984, p. 340, note Sousi.

42 Cass. com., 19 janvier $1988, n^{\circ} 85-18.443$, Bull.civ. IV, no 33, p. $23 ; J . C . P$. . d. G., 1988 , IV, p. 114 ; Rev. Soc. 1988 , p. 565 , note Castro.

43 Decret du 30 mai 1984 (J.O, 31 mai 1984 , p. 1699) relatif au registre du commerce et des sociétés.

44 Loi du 20 mars 1956 relative a la location gérance du fonds de commerce (article 4).

45 L'immatriculation ne s'adresse qu'aux commerçants, aux personnes physiques, aux sociétés commerciales ou civiles (sauf les sociétés en participation) et à certaines autres personnes morales dont l'immatriculation est prévue par les dispositions législatives ou réglementaires, (Article ler du Décret du 30 mai 1984). Aussi, doivent être immatriculées - et c'est une exception - les associations autorisées à êmettre des valeurs mobilières. Voir la Loi du 11 juillet 1985 (J.O. 12 juillet 1985).

46 Reinhard, Castro, Sousi.

47 Rep. Min., n* 1737, J.O.A.N.Q. 3 octobre $1988_{+}$p. 2751. Mais en 1992, la Cour de cassation persiste dans son interpretation en rejetant le pourvoi formé contre $\mathrm{l}^{\text {' }}$ arrêt de la Cour d'appel de Paris qui estimait que l'activité commerciale ne conférait pas à l'association la qualité de commerçant, que selon $1^{\prime}$ article $1^{\text {a }}$ du décret du 30 mai 1984, l'inscription au registre du commerce et des so- ciétés ne peut être accordée qu'aux personnes physiques - en raison de leur qualité de commerçant - et à certaines personnes morales déterminées par la loi et le règlement. Cass. Com. 01.03.94, $n^{\circ}$ 92-1513.529, La lettre des associations, $\mathrm{n}^{\circ}$ 55, 30 mars 1994.

48 Loi $\mathrm{n}^{\mathrm{n}} 67-563$ du 13 juillet 1967 : Loi du $1^{\text {ta }}$ mars 1984 sur la prévention des entreprises ; Loi du 10 juin 1994 relative à la prévention et au traitement des difficultes des entreprises.

49 Loi sur les compagnies, L.R.O., c. C-38; loi sur les corporations canadiennes, L.R.C., $\mathrm{c}$. C-32.

50 Articles $2831,2862,2854$ du Code civil du Québec sur la preuve en matiàre commerciale ; articles 1764,1767 concemant la vente de l'entreprise ; article 1525 sur la présomption de solidarité ; articles 2684 et 2685 sur une hypothèque concemant l'universalité de biens ou un meuble corporel représenté par un titre négociable...

51 Articles 1472 et 1612 du Code civil du Québec sur le secret commercial et les articles 2007,2014 a 2018 et 2021 concernant l"affrètement maritime.

52 Instruction fiscale relative aux associations, Bulletin officiel des impóts, 15 septembre 1998.

53 * The Gull Bay Development Corporation v. The Queen * . (1984) C.T.C., p. 159.84 D.T.C., p. 6040 .

\section{RÉFÉRENCES}

Bohémier, Albert et Côté, Pierre-Paul (1985), Droit commercial général, tome 1, Montréal, Thémis, 3" edition, 1985.

Castro, Sami (1987), Les associations exerçant une acrivité commerciale, thèse de doctorat en droit ( $3^{2}$ cycle), Paris, Université de Paris I.

Castro, Sami et Alix, Nicole (1990), L'entreprise associative - Aspects juridiques de l"intervention economique des associations, Paris, UNIOPSS-Économica.

Dandonneau, Antoni M. (1978), \& La francisation à I'aveuglette du droit des corporations *; R.J.T, vol. 13, n $\mathrm{n}^{\circ} 1$.

Ducharme, Léo (1976), De l'acte de commerce en droit québécois, thèse de doctorat, Université de Montréal.

L'Heureux, Nicole (1975), Précis de droit com: mercial au Québec, Québec, Les Presses de $1^{\prime}$ Université Laval, $2^{2}$ édition.

L'Heureux, Nicole (1986), Droit de la consom-

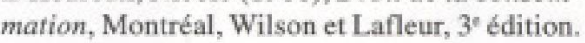

Martel, Paul, et Lebel, Georges A. (1994), La corporation sans but lucratif au Québec, tome 1. Montréal, Wilson et Lafleur/Martel Latic, Edition à jour.

Perreault , Antonio, (1936), Traité de droit commercial, tome 2, Montréal, Éditions Albert Levesque. 